\title{
RESPUESTA TÉRMICA DEL INVERNADERO DE LA ESTACIÓN EXPERIMENTAL FABIO BAUDRIT MORENO, ALAJUELA, COSTA RICA ${ }^{1}$
}

\author{
Alberto José López-López ${ }^{2}$, Carlos Benavides-León ${ }^{2}$
}

\section{RESUMEN}

Respuesta térmica del invernadero de la Estación Experimental Fabio Baudrit Moreno, Alajuela, Costa Rica. El objetivo de este trabajo fue simular la respuesta de la temperatura interna de un invernadero, con el fin de evaluar su comportamiento térmico en función de los requerimientos del cultivo. El modelo se validó con información de un periodo de cuatro meses segregado en dos intervalos (diciembre 2009 y agosto, setiembre y octubre 2010). Este considera la interacción de los cultivos, así como las condiciones ambientales externas como radiación, temperatura, humedad relativa, velocidad y dirección del viento. La temperatura interna se comparó con la óptima recomendada en la literatura para chile dulce (Capsicum annuum), tomate (Solanum lycopersicum) y melón (Cucumis melo). Los resultados obtenidos presentan un valor de la raíz del error medio cuadrático relativo para la temperatura simulada de $2,99 \%$ y $4,69 \%$ para cada intervalo estudiado. Esto demuestra que es posible predecir la respuesta térmica del invernadero y si su localización, diseño y aditamentos operativos son adecuados para el o los cultivos deseados. La metodología permitió evaluar las instalaciones para el desarrollo de diferentes cultivos, además, es aplicable para evaluar la factibilidad técnica de implementar mejoras en el diseño y el equipamiento, para alcanzar las condiciones óptimas requeridas por los cultivos específicos. Se concluye que el invernadero, bajo las condiciones actuales de operación, es adecuado para el cultivo del melón, en menor condición para el chile y del todo no es recomendable, en ciertas épocas del año, para el tomate.

Palabras clave: ambientes protegidos, control climático en ambientes protegidos, selección de cultivos en ambientes protegidos modelación climática.

\begin{abstract}
Thermal behavior of a greenhouse located in Fabio Baudrit Moreno Experimental Station, Alajuela, Costa Rica. The following study presents a simulation of the temperature inside a greenhouse, in order to evaluate thermal behavior according to crop requirements. The model was calibrated using climatic data during four months divided in two intervals (December 2009 and August, September and October 2010) and validated the interactions between the greenhouse's atmospheric conditions, the crops requirements and the external climatic factors such as radiation, temperature, relative humidity, wind speed and direction. The internal temperature was compared with the optimal recommended by the literature for different crops, i.e. sweet pepper (Capsicum annuum), tomato (Solanum lycopersicum) and melon (Cucumis melo). The results generated a relative root medium square error of $2.99 \%$ and $4.69 \%$ for the simulated temperature in two different intervals. This showed that it is possible to predict the thermal behavior of the greenhouse, and if the location, overall design and climate control equipment are suitable for the chosen crops. The methodology also allowed the assessment for different type of crops within the protected environment and may be applied to evaluate the technical feasibility to implement improvements in the design and equipment selection, in order to achieve the optimum conditions required for the specific crops. It was concluded that the facility is suitable to produce melon and sweet pepper to a lesser degree, and was not recommended for tomato production during certain months.
\end{abstract}

Keywords: protected environments, climate control in protected environments, selection of crops in protected environments, steady modeling.

\footnotetext{
1 Recibido: 2 de julio, 2013. Aceptado: 1 de abril, 2014. Investigación generada como parte del proyecto "Evaluación del comportamiento de los parámetros térmicos y estructurales que rigen el diseño de invernaderos para su adaptación a las condiciones agroclimáticas en el país", proyecto No. B 0248-041 inscrito en la Vicerrectoría de Investigación de la Universidad de Costa Rica en el año 2010.

2 Escuela de Ingeniería Agrícola, Universidad de Costa Rica. San José, Costa Rica. ajllaj@ucr.ac.cr, carlos.benavidesleon@ucr.ac.cr
} 


\section{INTRODUCCIÓN}

Un invernadero en una estructura que se construye con el fin de asegurar un ambiente óptimo para el desarrollo del cultivo y controlar plagas. Según el grado de dominio que se ejerza sobre las condiciones ambientales como temperatura, humedad relativa, dióxido de carbono, radiación, luz, velocidad de viento, características de la construcción, los materiales y el equipamiento, puede operar como un ambiente protegido o como un ambiente controlado.

Autores como Leal y Costa (2011), Alas (2003), y Barquero (2001), coinciden en que la eficiencia de la inversión en un invernadero, asumiendo un correcto manejo agronómico del cultivo, estará en función de un adecuado diseño y operación de la instalación, para lo cual debe controlarse la interacción de las condiciones ambientales con la precisión que el cultivo requiera.

La modelación del clima en invernaderos es ampliamente estudiada en el mundo, con el fin de aumentar el crecimiento y productividad de los cultivos. Lo que se busca es predecir la respuesta climática de la instalación, representada básicamente por la temperatura y la humedad relativa, para que el cultivo por desarrollar lo haga en su ambiente óptimo.

Estudios como el de Stanghellini (1987) presentan la transpiración como una ayuda al manejo del clima en el invernadero. El efecto en el enfriamiento del sombreo, la ventilación y la transpiración, actuando conjuntamente, son evaluados por Kittas (2003), para regular el clima interno con el fin de obtener cosechas óptimas. La contribución de la transpiración como forma de enfriamiento evaporativo es estudiada por Montero (2006) y Piscia et al. (2012), quienes analizaron el efecto de diferentes formas de sombreo y ventilación en invernaderos españoles, para disminuir la temperatura interna. Vanthoor (2011) propuso una metodología de diseño de agricultura protegida, aplicable a nivel mundial, basada en el clima y las condiciones económicas locales, tendiente a definir técnicas que puedan influenciar el clima interno. Para efectos de modelar la producción de tomate en función de la temperatura, este investigador cita varios estudios que relacionan la producción con temperaturas superiores a las óptimas, como Adams et al. (2001), quienes reportaron que a una temperatura media de $26^{\circ} \mathrm{C}$, se obtuvo una producción 18\% mayor de la observada a $22^{\circ} \mathrm{C}$; Peet et al. (1997) encontraron a una temperatura media de $29^{\circ} \mathrm{C}$, una cosecha superior en $17 \%$ respecto a la observada a $25^{\circ} \mathrm{C}$; sin embargo, Zhang et al. (2008) observaron a una temperatura de $35^{\circ} \mathrm{C}$ una caída en la cosecha a 46,1\% comparado con una temperatura de $25^{\circ} \mathrm{C}$. Aparte de la caída en el rendimiento de la cosecha, se citan otros efectos como menor cuaje de la fruta, menor periodo de crecimiento del fruto, frutos más pequeños y con menor peso. También se citan efectos relacionados con bajas temperaturas, variaciones de esta por cortos y largos periodos y por diferencias en la presión de vapor. Estas investigaciones demuestran la importancia de considerar la temperatura y otras variables climáticas que operan en el ambiente protegido, para lograr sacar mayor provecho a la inversión.

De acuerdo con Tesi (2001), para todo cultivo existen diferentes rangos de luz, radiación, temperatura y humedad relativa durante el día y la noche, en las diferentes etapas de su desarrollo fenológico, que le permite optimizar su desarrollo foliar, la floración y la productividad. Autores como Castellanos (2009), Leal y Costa (2011), Serrano (1994), Tesi (2001) definen las temperaturas y humedades relativas óptimas de diversos cultivos.

En la investigación de literatura realizada no se encontraron artículos que analicen la operación de ambientes protegidos en el país, relacionada con las características climáticas del ambiente interno. Por esta razón, se realizó una investigación aplicada a un invernadero que permita evaluar y predecir si la instalación tiene un diseño térmico apropiado y su operación climática es óptima. El control de variables como la temperatura y la humeda relativa es parte del reto, para que la inversión de construir una instalación del tipo en estudio sea rentable y contribuya a mejorar la competitividad de nuestros agricultores.

El invernadero analizado es empleado para investigación, razón por la cual debe ser capaz de operar en un rango más amplio que invernaderos para cultivos específicos, y permitir el cultivo simultáneo de diferentes variedades.

El objetivo de este trabajo fue simular la respuesta de la temperatura interna de un invernadero, con el fin de evaluar su comportamiento térmico en función de los requerimientos del cultivo. 


\section{MATERIALES Y MÉTODOS}

El análisis fue realizado entre los meses de febrero y agosto del 2012 utilizando información facilitada por el Instituto Meteorológico Nacional correspondiente al periodo de mayo 2009 a octubre 2011. Este se llevó a cabo en un invernadero ubicado en la Estación Experimental Agrícola Fabio Baudrit Moreno (EEFBM), latitud $10^{\circ} 0$ ' $22,89^{\prime \prime} \mathrm{N}$ y longitud $84^{\circ} 15^{\prime} 54,63$ 'O a 840 $\mathrm{msnm}$. El invernadero se orienta en sentido este-oeste de acuerdo con la Figura 1.

Este es un invernadero multicapilla de $55 \mathrm{~m}$ de largo y $38,4 \mathrm{~m}$ de ancho, con cuatro naves adosadas, una altura a la canoa de $4 \mathrm{~m}$ y al cenit de $6,5 \mathrm{~m}$. Las ventanas en los costados este y oeste tienen un área total de $206,9 \mathrm{~m}^{2}$ y las ventanas en dirección norte y sur tienen un área de $220 \mathrm{~m}^{2}$. Las ventanas cenitales, en su máxima apertura, tienen una altura de $2,1 \mathrm{~m}$.

Se evaluó el comportamiento interno de la temperatura y la humedad relativa del invernadero con información suministrada por el Departamento de Hortalizas de la Estación Experimental Fabio Baudrit Moreno para dos periodos: el primero, durante el mes de diciembre 2009 y el segundo, agosto, setiembre y octubre 2010.

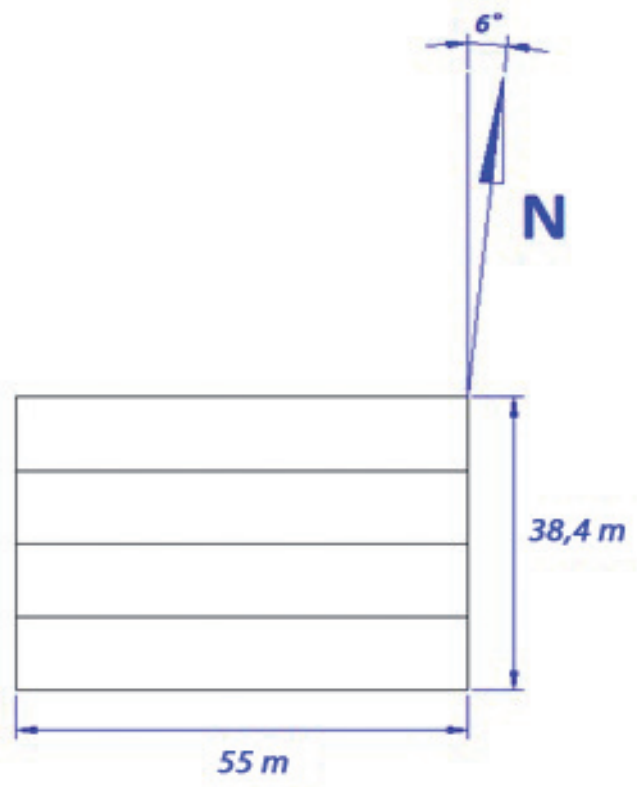

Figura 1. Orientación del invernadero. Estación Experimental Fabio Baudrit Moreno (EEFBM), Alajuela, Costa Rica. Febrero 2012.
El comportamiento real del invernadero en términos de temperatura, en el mes de diciembre del 2009 (inicio de la época seca), así como durante los meses de agosto, setiembre y octubre del 2010, se obtuvo con las mediciones de temperatura y humedad relativa en intervalos de 15 minutos y se utilizaron promedios de intervalos de las 07:00 a las 17:00 horas del día, con base en información suministrada por la EEFBM. Esta información fue utilizada para comprobar la respuesta del modelo.

Además se evaluó la dirección del viento predominante y la velocidad media en dicha dirección, con un análisis de frecuencia diaria. Se categorizó la serie de datos de intervalo horario obtenidas por el sensor de dirección de viento de la estación meteorológica, en ocho direcciones definidas por rangos de ángulos consecutivos de $45^{\circ}$. Se asignó así la velocidad a la dirección de viento correspondiente a esa hora. Se sumaron los datos de direcciones de viento horario, para obtener el total de veces que el sensor registró cada una de las ocho diferentes direcciones definidas.

Se calculó el porcentaje de cada una de las direcciones para cada día del mes y se estableció la dirección predominante diaria de viento como la dirección de porcentaje más alto. Seguidamente, se promediaron los datos de velocidad de viento horario para esa dirección, con lo que se obtuvo la velocidad media diaria en la dirección predominante.

Se obtuvo el análisis de frecuencia mensual utilizando la información de dirección diaria de viento y el promedio mensual de la velocidad.

Además, se realizó una investigación incluyendo varios autores (Serrano, 1994; Tesi, 2001; Castellanos, 2009; Leal y Costa, 2011, entre otros) para definir las temperaturas y humedades relativas óptimas de diversos cultivos. Se definió la temperatura y humedad relativa óptimas del ambiente interno del invernadero siguiendo las recomendadas por Tesi (2001), para los cultivos de chile dulce (Capsicum annuum), tomate (Solanum lycopersicum) y melón (Cucumis melo), ya que estos son los cultivos que con más frecuencia se desarrollan en el invernadero en estudio.

Se aplicó un modelo termodinámico estático basado en las ecuaciones de balance de energía de Albright (1990), Hellickson (1983) y Leal y Costa (2011). Para predecir la temperatura interna, se requirieron promedios externos de temperaturas, humedad relativa, radiación, dirección y velocidad 
de viento. Otras constantes físicas relacionadas con características de los materiales, se obtuvieron en las mismas referencias.

El modelo termodinámico estático utilizado se define por la siguiente ecuación:

$$
Q_{s o}+Q_{r e s p}+Q_{v e}=Q_{f o t}+Q_{c e}+Q_{s p}+Q_{s l}+Q_{v s}+Q_{t}
$$

$\mathrm{Q}_{\mathrm{so}}$ : Calor sensible de radiación solar, $\mathrm{W}$.

$\mathrm{Q}_{\mathrm{fot}}$ : Calor sensible usado para la fotosíntesis, W.

$\mathrm{Q}_{\text {resp }}$ : Calor sensible de respiración del cultivo, $\mathrm{W}$.

$\mathrm{Q}_{\mathrm{ve}}$ : Calor sensible de aire de ventilación de entrada, W. W.

$\mathrm{Q}_{\mathrm{ce}}$ : Calor sensible de conducción de la estructura,

$\mathrm{Q}_{\mathrm{sp}}$ : Calor sensible transferido al suelo o piso por el perímetro, $\mathrm{W}$.

$\mathrm{Q}_{\mathrm{sl}}$ : Calor sensible convertido en calor latente dentro del espacio interno, $\mathrm{W}$.

$\mathrm{Q}_{\mathrm{sv}}$ : Calor sensible de aire de ventilación de salida, W.

$\mathrm{Q}_{\mathrm{tt}}$ : Calor de irradiación o de transmitancia térmica, W.

El detalle de las ecuaciones se discute en López (2013). Los valores de las diferentes variables que se utilizaron como parte del modelo para el balance de energía se presentan en el Cuadro 1. Para cada valor de la temperatura interna que se deseaba simular, se requirió accesar como entrada, los valores promedios de temperaturas, humedad relativa y radiación externas; además de dirección y velocidad de viento.

El valor de área de abertura corresponde al de la ventana ubicada en el costado este del invernadero, por ser la dirección del viento predominante, según análisis previos. Las dimensiones del invernadero se midieron en el lugar. El área y longitud de arco se calculó con el programa AutoCAD. El valor de emisividad del suelo se tomó como 0,95 , por las propiedades térmicas y ópticas del material. La densidad del aire se calculó para cada análisis individual por medio de ecuaciones psicrométricas.

Para comprobar la precisión del modelo, se calculó la respuesta del invernadero utilizando la información meteorológica del periodo del cual se brindaron las condiciones ambientales y mediciones del clima interno obtenidas por el Departamento de Hortalizas de la Estación Experimental Fabio Baudrit Moreno.

Con el fin de predecir el comportamiento climático interno del invernadero del año completo con respecto a
Cuadro 1. Valores utilizados en el balance energético en un invernadero de la Estación Experimental Agrícola Fabio Baudrit Moreno, Alajuela, Costa Rica. Marzo 2012.

\begin{tabular}{lc}
\hline Datos del invernadero & \\
\hline Altitud (msnm) & 840 \\
\# de naves & 4 \\
Largo de naves (m) & 55 \\
Ancho de naves (m) & 9,6 \\
Área arco ( ${ }^{2}$ ) & 13,3 \\
Long. arco (m) & 10,7 \\
Alto a canoa (m) & 4 \\
Alto al cenit (m) & 6,5 \\
F: factor perimetral, W/m K & 1,15 \\
Largo total (m) & 55 \\
Ancho total (m) & 38,4 \\
Perímetro (m) & 186,8 \\
Área de cubierta: Ac ( ${ }^{2}$ ) & 2351,6 \\
Área de piso (m ${ }^{2}$ ) & 2112 \\
\hline Datos cubierta & \\
Transmitancia de onda corta (adimensional) & $0,8^{*}$ \\
Transmitancia de onda larga (adimensional) & $0,75^{*}$ \\
Coeficiente global de transferencia de calor: U (W/ & \\
$\mathrm{m}^{20} \mathrm{C}$ ) & $7,14^{*}$ \\
\hline Datos aberturas & 206,9 \\
Área de aberturas Aa: ( ${ }^{2}$ ) & 0,35 \\
Eficiencia de aberturas: E (adimensional) & \\
\hline
\end{tabular}

*Información suministrada por la empresa proveedora de la cubierta, Olefinas de Costa Rica S.A.

los cultivos sembrados con más frecuencia, se utilizaron factores de cobertura de 0,5 y 1 correspondientes al invernadero cultivado a un $50 \%$ y el invernadero cultivado en su totalidad respectivamente.

Se formularon distintos escenarios, usando las temperaturas máximas promedio y las absolutas mensuales de los meses de abril y agosto con información de IMN (2012), por ser estos los meses de condiciones de temperatura y velocidad de viento externas críticas.

Se utilizaron porcentajes de $20 \%$ y $50 \%$ de área del invernadero cultivadas, que responden a la realidad en el momento de la investigación. Además, se utilizaron temperaturas de $23^{\circ} \mathrm{C}$ y $25^{\circ} \mathrm{C}$, respectivamente, como límites inferior y superior de temperatura deseados dentro del invernadero, esto debido a que esas temperaturas se encuentran en un 
rango óptimo para los cultivos más comunes en la instalación estudiada.

\section{RESULTADOS Y DISCUSIÓN}

\section{Análisis de las condiciones climáticas ambientales}

Los meses con condiciones críticas para el periodo analizado son abril, por poseer los promedios de temperaturas máximas más altas, y agosto, por tener los promedios de velocidades de viento más bajas. Se evidenció un cambio en la dirección del viento predominante en los meses de junio, septiembre y octubre (Cuadro 2).

El periodo con mayor velocidad de viento promedio estuvo comprendido entre los meses de diciembre a marzo. Los meses con menores velocidades presentaron mayor variación en la dirección. Los resultados del análisis del viento predominante en el mes de marzo (mes más ventoso) se presenta en la Figura 2, como ejemplo del análisis realizado.

\section{Necesidades climáticas de los cultivos sembrados con más frecuencia en el invernadero estudiado}

La temperatura adecuada del aire dentro del invernadero se da en función de los niveles óptimos

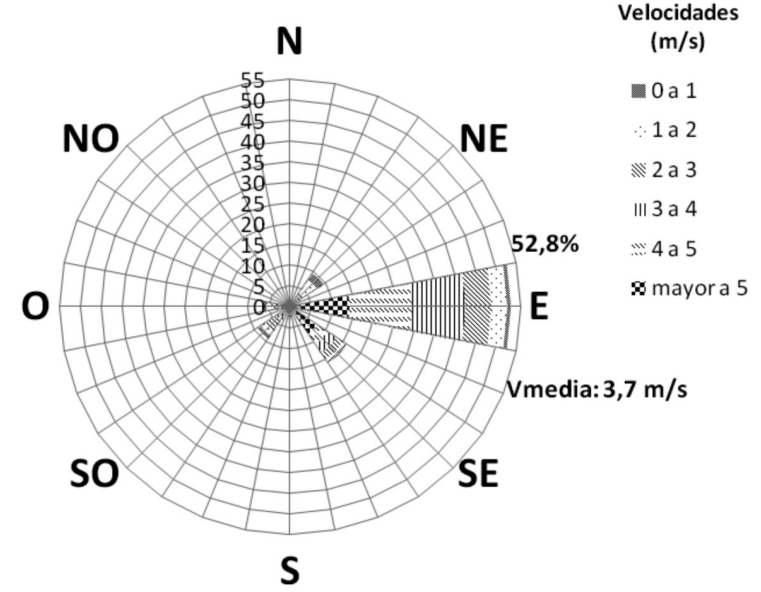

Figura 2. Frecuencia y distribución de velocidades y dirección de viento para el mes de marzo. Estación Experimental Agrícola Fabio Baudrit Moreno. Marzo 2012.

Fuente: Elaboración propia con información de Instituto Meteorológico Nacional (IMN, 2012).

para el crecimiento de los diferentes cultivos del invernadero. Según Castellanos (2009), el rango óptimo de crecimiento y producción de tomate se da entre los 15 y $25^{\circ} \mathrm{C}$; el rango térmico óptimo para el desarrollo del tomate durante el día se encuentra entre $23-25^{\circ} \mathrm{C}$, mientras que la humedad relativa apropiada

Cuadro 2. Promedios mensuales obtenidos de información climática horaria de la estación meteorológica ubicada en la Estación Experimental Agrícola Fabio Baudrit Moreno, Alajuela, Costa Rica. Periodo mayo 2009 a octubre 2011.

\begin{tabular}{|c|c|c|c|c|c|c|c|c|c|c|c|c|}
\hline \multirow{2}{*}{$\begin{array}{l}\text { Estación Experimental } \\
\text { Fabio Baudrit Moreno } \\
\text { Datos mensuales } \\
\end{array}$} & \multicolumn{4}{|c|}{$\begin{array}{c}\text { Altitud } \\
840 \mathrm{msnm}\end{array}$} & \multicolumn{4}{|c|}{$\begin{array}{c}\text { Lat. } \\
10^{\circ} 0{ }^{\prime} 22.89{ }^{\prime} \mathrm{N}\end{array}$} & \multicolumn{4}{|c|}{$\begin{array}{c}\text { Long. } \\
84^{\circ} 155^{\prime} 54.63 \text { 'O }\end{array}$} \\
\hline & ENE & FEB & MAR & ABR & MAY & JUN & JUL & AGO & SEP & OCT & NOV & DIC \\
\hline TMP $\left({ }^{\circ} \mathrm{C}\right)$ & 28,6 & 29,5 & 30 & 30,1 & 28,5 & 27,6 & 27,5 & 27,9 & 27,6 & 26,3 & 26,5 & 27,4 \\
\hline D.E & 1,3 & 1,3 & 1,6 & 1,5 & 1,6 & 1,4 & 2,0 & 1,7 & 2,3 & 2,6 & 1,7 & 2,0 \\
\hline $\operatorname{HR} . \mathrm{mP}(\%)$ & 40,9 & 40,9 & 37,9 & 42,3 & 54,7 & 60,6 & 59,1 & 57,3 & 60 & 64,4 & 59,3 & 47,4 \\
\hline D.E & 6,5 & 7,0 & 6,6 & 10,2 & 9,7 & 7,3 & 10,5 & 9,4 & 11,2 & 13,3 & 10,6 & 8,8 \\
\hline $\mathrm{RP} *\left(\mathrm{~W} / \mathrm{m}^{2}\right)$ & 623 & 649 & 672 & 636 & 582 & 551 & 533 & 569 & 593 & 556 & 548 & 588 \\
\hline D.E & 61,6 & 99,5 & 58,5 & 142,3 & 149,1 & 138,8 & 163,1 & 146,2 & 146,8 & 164,2 & 157,3 & 116,0 \\
\hline $\operatorname{VVP}(\mathrm{m} / \mathrm{s})$ & 3,6 & 3,6 & 3,7 & 2,6 & 2,1 & 2,4 & 1,9 & 1,7 & 2,3 & 2,1 & 2,2 & 3,3 \\
\hline D.E & 1,2 & 1,3 & 1,2 & 0,9 & 1,2 & 0,5 & 1,1 & 0,7 & 0,6 & 0,6 & 1,1 & 1,2 \\
\hline DVP & $\mathrm{E}$ & $\mathrm{E}$ & $E$ & $\mathrm{E}$ & $\mathrm{E}$ & SO & $E$ & $\mathrm{E}$ & SO & SO & $\mathrm{E}$ & $E$ \\
\hline
\end{tabular}

D.E: Desviación estándar; TMP: temperatrura máxima promedio; HR.mP: Humedad relativa mínima promedio; RP: Radiación promedio; VVP: Velocidad de viento promedio en la dirección predominante; DPV: Dirección predominante de viento.

* Se tomaron los valores de radiación máxima horaria entre las 07:00 y las 17:00 horas.

Fuente: Elaboración propia con información de IMN (2012). 
es del orden de 70\%. Según Leal y Costa (2011), las temperaturas óptimas para el crecimiento del tomate es de $18^{\circ} \mathrm{C}$ a $21^{\circ} \mathrm{C}$, para el chile de $20^{\circ} \mathrm{C}$ a $25^{\circ}$ y para el melón de 25 a $30^{\circ} \mathrm{C}$. Las humedades relativas óptimas se encuentran entre 50 y $80 \%$ para estos cultivos.

Los rangos propuestos por Tesi (2001) se relacionan con los rangos antes mencionados como se observa en el Cuadro 3.

La humedad relativa del aire dentro del invernadero interviene en la transpiración, en el crecimiento de los tejidos, en la fecundación de las flores, y en el desarrollo de enfermedades (Serrano, 1994). Los valores de humedad relativa del aire adecuados para los cultivos del invernadero se encuentran arriba de 55\% (Cuadro 4).

Para el mes de diciembre del 2009, la temperatura promedio diurna interna, en la mayor parte del intervalo, se encontraba entre el límite interior del rango óptimo del melón y el límite superior del rango óptimo del chile; en ocasiones presentando temperaturas superiores a las óptimas para chile y el tomate, para ese mes (Figura 3). En la mayor parte de este periodo, la humedad relativa se mantuvo por debajo de los límites óptimos inferiores de los cultivos (Figura 4).

Para el intervalo correspondiente entre los meses de agosto y octubre del 2010, los valores de temperatura interna del invernadero se mantuvieron entre el límite superior óptimo del chile y del tomate (Figura 3). En los últimos días del mes de setiembre, se pudo observar una disminución de las temperaturas por debajo de $\operatorname{los} 24^{\circ} \mathrm{C}$. Las humedades relativas internas se mantuvieron entre $60 \%$ y $70 \%$ en los primeros 35 días del intervalo. A finales del mes de setiembre, se observó un incremento de la humedad relativa, durante un periodo corto llegando a valores superiores a $85 \%$. Se observaron leves disminuciones a mediados del mes de octubre. La humedad relativa para estos meses se encontró durante la mayor parte del tiempo en los
Cuadro 4. Valores de humedad relativa del aire adecuados para el cultivo de melón, chile y tomate en invernadero. Estación Experimental Agrícola Fabio Baudrit Moreno, Alajuela, Costa Rica. Marzo 2012.

\begin{tabular}{lc}
\hline Cultivo & $\begin{array}{c}\text { Humedad relativa óptima del } \\
\text { aire para el crecimiento de } \\
\text { cultivo en invernadero (\%) }\end{array}$ \\
\hline $\begin{array}{l}\text { Melón (Cucumis melo) } \\
\text { Chile (Capsicum annuum) } \\
\text { Tomate }\end{array}$ & 60 a 80 \\
(Solanum lycopersicum) & 65 a 70 \\
\hline
\end{tabular}

Fuente: Tesi (2001).

intervalos óptimos de los cultivos de melón y tomate (Figura 6).

\section{Simulación de temperatura interna del invernadero}

Al comparar los promedios de las temperaturas internas del invernadero del mes de diciembre del 2009 , medidas con las simulaciones realizadas con el modelo estático, utilizando un factor de cultivo de 0,5 y una velocidad promedio de viento en dirección este, se obtuvieron dferencias promedio de $0,75^{\circ} \mathrm{C}$ y un porcentaje de la raíz del error medio cuadrático relativo (REMCR) de 4,69. Se obtuvieron diferencias altas de $2,91^{\circ} \mathrm{C}, 2,15^{\circ} \mathrm{C}$ y $3,07^{\circ} \mathrm{C}$ en tres días atípicos, con poca velocidad de viento y valores pequeños de radiación solar. En el resto de los días, la variación entre la temperatura interna medida y simulada fue menor a $1,6^{\circ} \mathrm{C}$ (Figura 7).

En la comparación de los promedios de las temperaturas internas del invernadero en los meses de agosto, setiembre y octubre de 2010, medidas con las simulaciones realizadas con el modelo estático, utilizando un factor de cultivo de 0,8 y una velocidad promedio de

Cuadro 3. Valores de temperatura adecuados para el cultivo de melón, chile y tomate en invernadero. Estación Experimental Agrícola Fabio Baudrit Moreno, Alajuela, Costa Rica. Marzo 2012.

\begin{tabular}{lccccc}
\hline Cultivo & $\begin{array}{c}\text { Temp. mínima } \\
\text { letal }\left({ }^{\circ} \mathbf{C}\right)\end{array}$ & $\begin{array}{c}\text { Temp. mínima } \\
\text { biológica }\left({ }^{\circ} \mathbf{C}\right)\end{array}$ & $\begin{array}{c}\text { Temp. óptima } \\
\text { día }\left({ }^{\circ} \mathbf{C}\right)\end{array}$ & $\begin{array}{c}\text { Temp. óptima } \\
\text { noche }\left({ }^{\circ} \mathbf{C}\right)\end{array}$ & $\begin{array}{c}\text { Temp. máxima } \\
\text { biológica }\left({ }^{\circ} \mathbf{C}\right)\end{array}$ \\
\hline Melón (Cucumis melo) & 0 & 12 a 14 & 24 a 30 & 12 a 14 & 30 a 34 \\
Chile (Capsicum annuum) & $(-2)$ a 0 & 10 a 12 & 22 a 28 & 16 a 18 & 28 a 32 \\
Tomate (Solanum lycopersicum) & $(-2)$ a 0 & 8 a 10 & 22 a 26 & 13 a 16 & 26 a 30 \\
\hline
\end{tabular}

Fuente: Tesi (2001). 


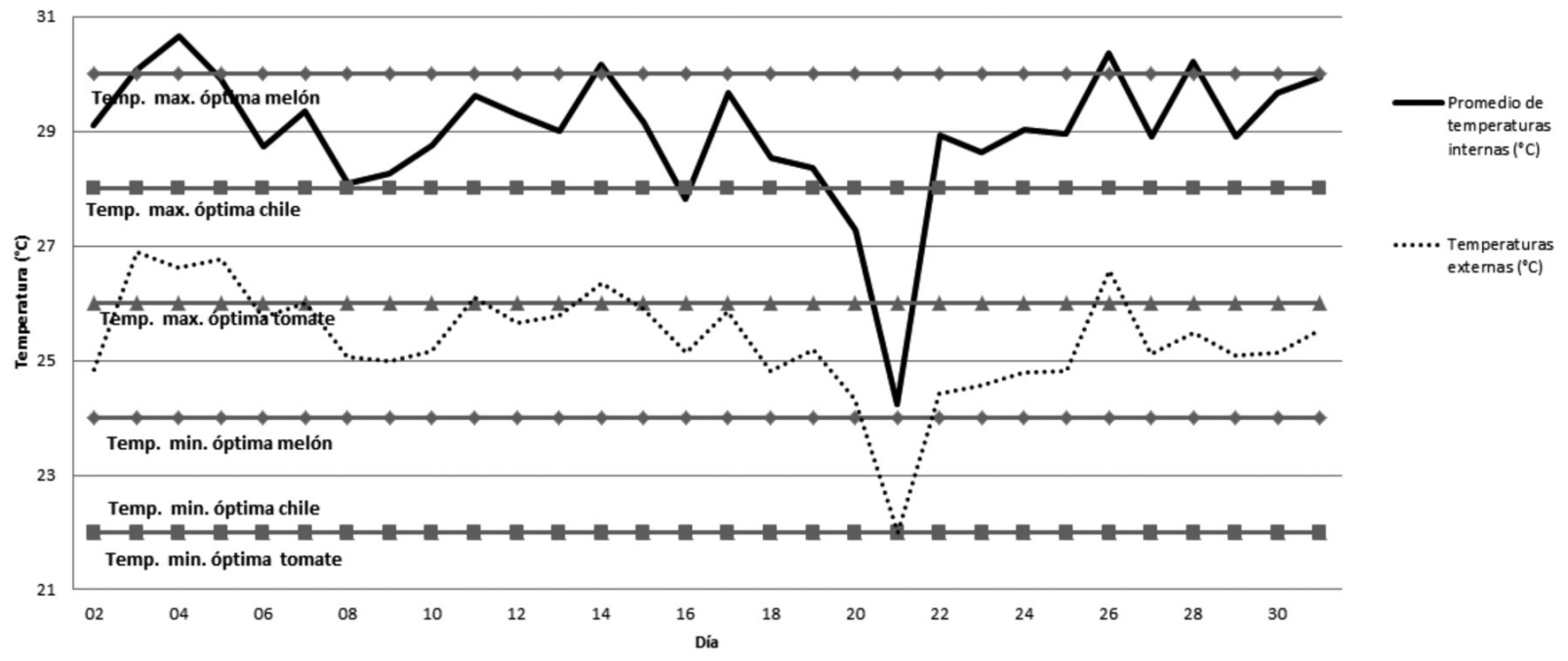

Figura 3. Temperaturas internas diurnas en diciembre del 2009 en el invernadero de la Estación Experimental Agrícola Fabio Baudrit Moreno y rango de temperaturas óptimas para melón, chile y tomate. Alajuela, Costa Rica. Mayo 2012. Fuente: Elaboración propia con información del IMN (2012).

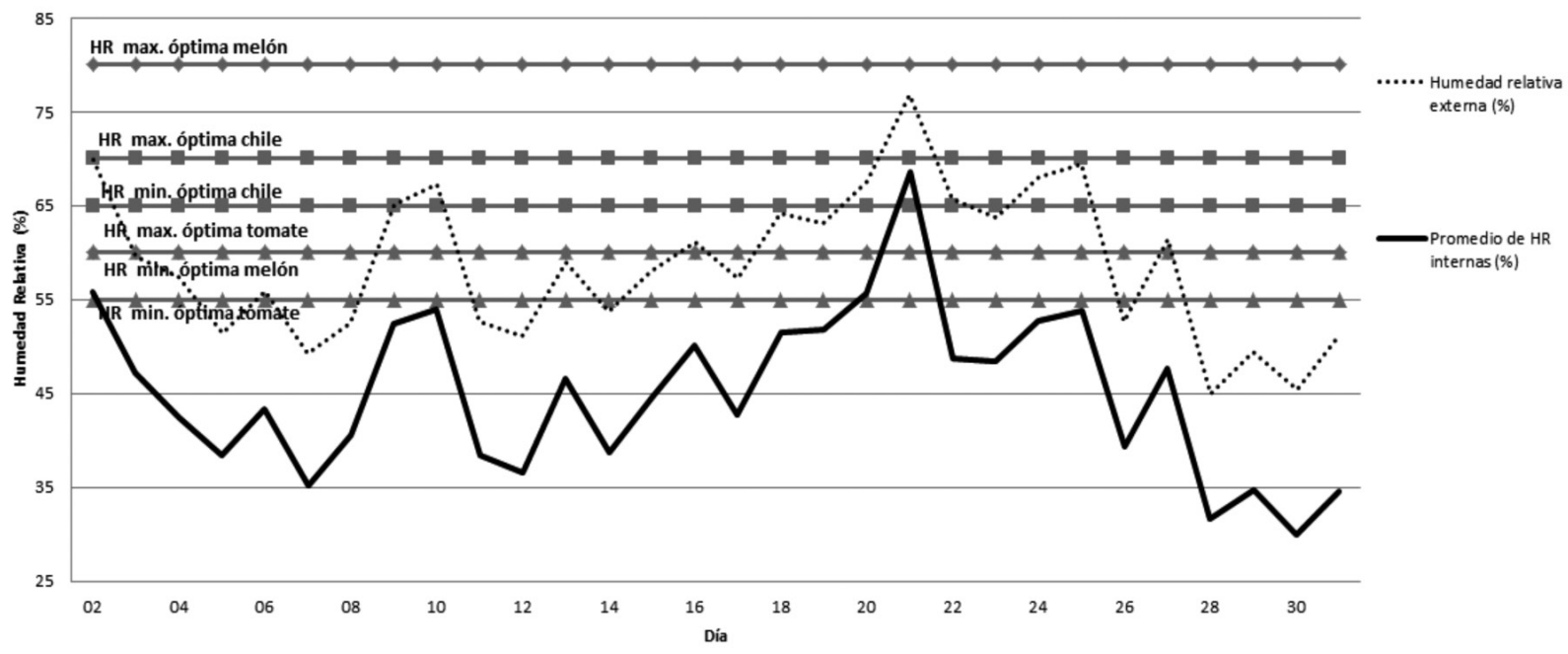

Figura 4. Humedades relativas internas diurnas en diciembre del 2009 en el invernadero de la Estación Experimental Agrícola Fabio Baudrit Moreno, y rango de humedades relativas óptimas, para melón, chile y tomate. Alajuela, Costa Rica. Mayo 2012. Fuente: Elaboración propia con información del IMN (2012).

viento en dirección suroeste, se obtuvieron diferencias promedio para el intervalo de $0,20^{\circ} \mathrm{C}$ y un REMCR de 2,99. La mayor diferencia diaria obtenida entre las temperaturas comparadas fue de $1,66^{\circ} \mathrm{C}$. Se observó que las temperaturas medidas fueron muy similares a las temperaturas simuladas (Figura 8). En estudios realizados por Costa et al. (2004), se observan diferencias promedio entre valores de temperatura simulados y 


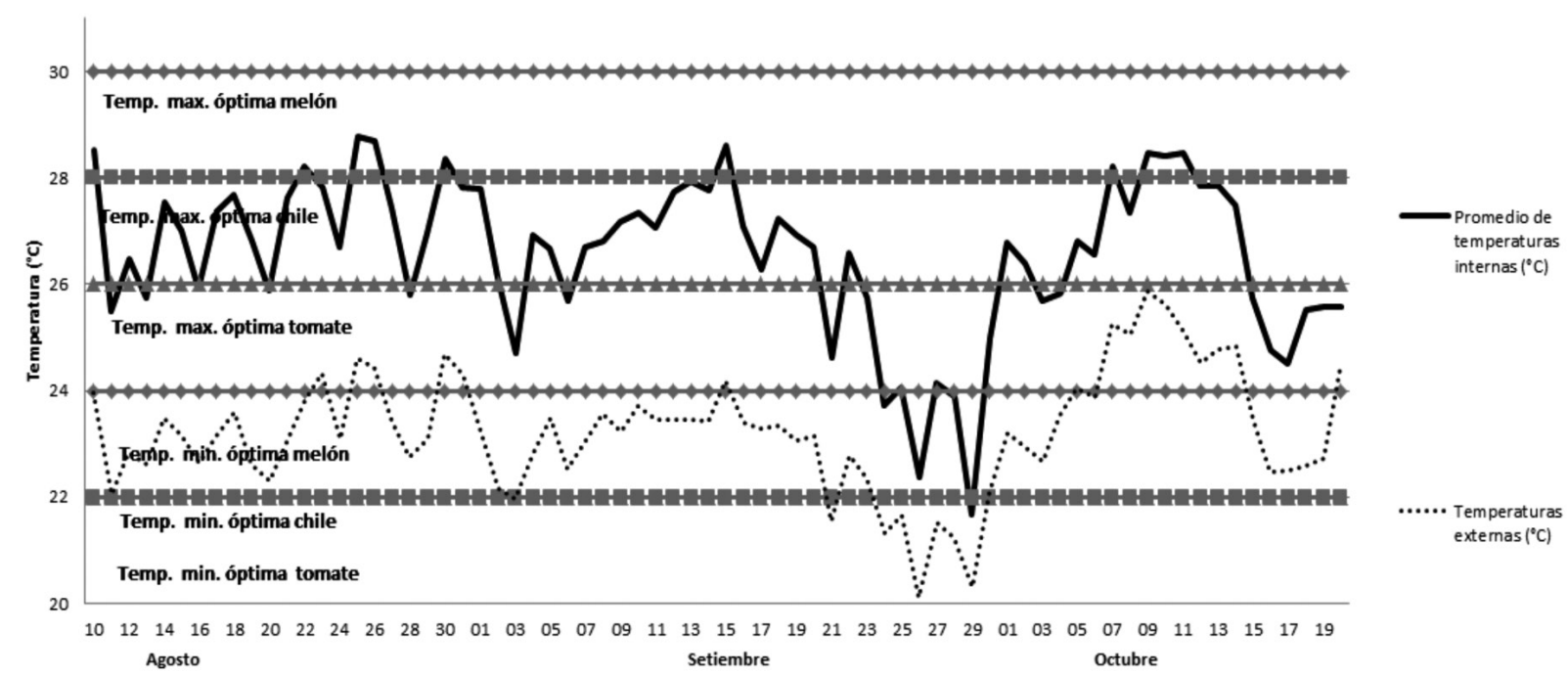

Figura 5. Temperaturas internas diurnas en los meses de agosto a octubre del 2010 en el invernadero de la Estación Experimental Agrícola Fabio Baudrit Moreno, y rango de temperaturas óptimas, para melón, chile y tomate. Alajuela, Costa Rica. Mayo 2012.

Fuente: Elaboración propia con información del IMN (2012).

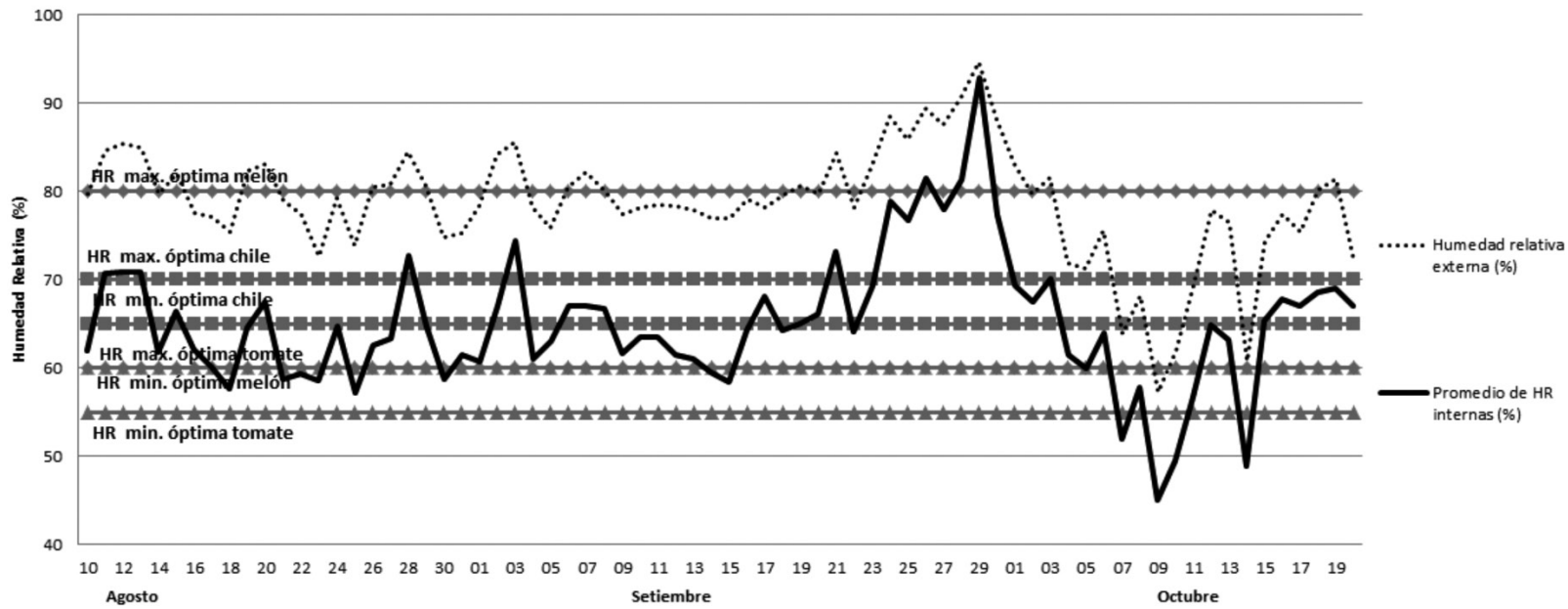

Figura 6. Humedades relativas internas diurnas en los meses de agosto a octubre del 2010 en el invernadero de la Estación Experimental Agrícola Fabio Baudrit Moreno y rango de humedades relativas óptimas para melón, chile y tomate. Alajuela, Costa Rica. Mayo 2012.

Fuente: Elaboración propia con información de IMN (2012).

observados de de $0,64^{\circ} \mathrm{C}$, lo cual es considerado acorde con los resultados de este estudio.

De acuerdo con el Cuadro 5, Vanthoor (2011), reportó valores de REMCR entre 3 y 8 en diez localidades para la simulación de temperatura en invernaderos, realizada con la técnica de modelación de Flujo Dinámico Computacional, lo cual ubica los valores encontrados en este estudio dentro del rango. 


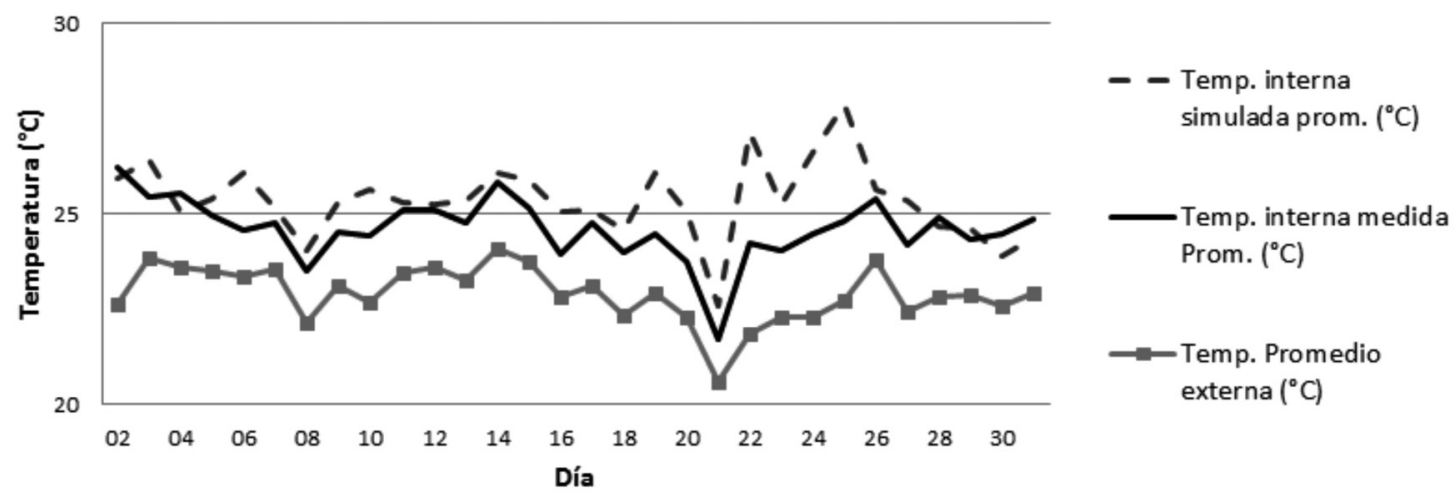

Figura 7. Temperaturas medidas y simuladas del invernadero de la Estación Experimental Agrícola Fabio Baudrit Moreno, en el mes de diciembre 2009. Alajuela, Costa Rica. Junio 2012.

Al simular el clima del invernadero para el año completo y con dos porcentajes de área cultivada, se observó que entre los meses de enero a setiembre, con un $50 \%$ de área cultivada, el invernadero no pudo alcanzar los rangos óptimos de los tres cultivos considerados, bajo los métodos de administración climática en que se encuentra (Figura 9). Al cultivar la totalidad del invernadero, se observó que el rango óptimo de temperaturas sólo se alcanza al cultivar melón en los meses de junio a diciembre. Esta diferencia en la temperatura interna se explica por el aumento de la conversión de calor sensible a calor latente que se genera con la transpiración de mayor área de cultivo.

Este estudio mostró que la simulación de la temperatura en un invernadero es posible mediante el uso de modelos estáticos. Esta herramienta se podría implementar en el país para el diseño de este tipo de instalaciones y así evaluar la respuesta climática previa a realizar una inversión.

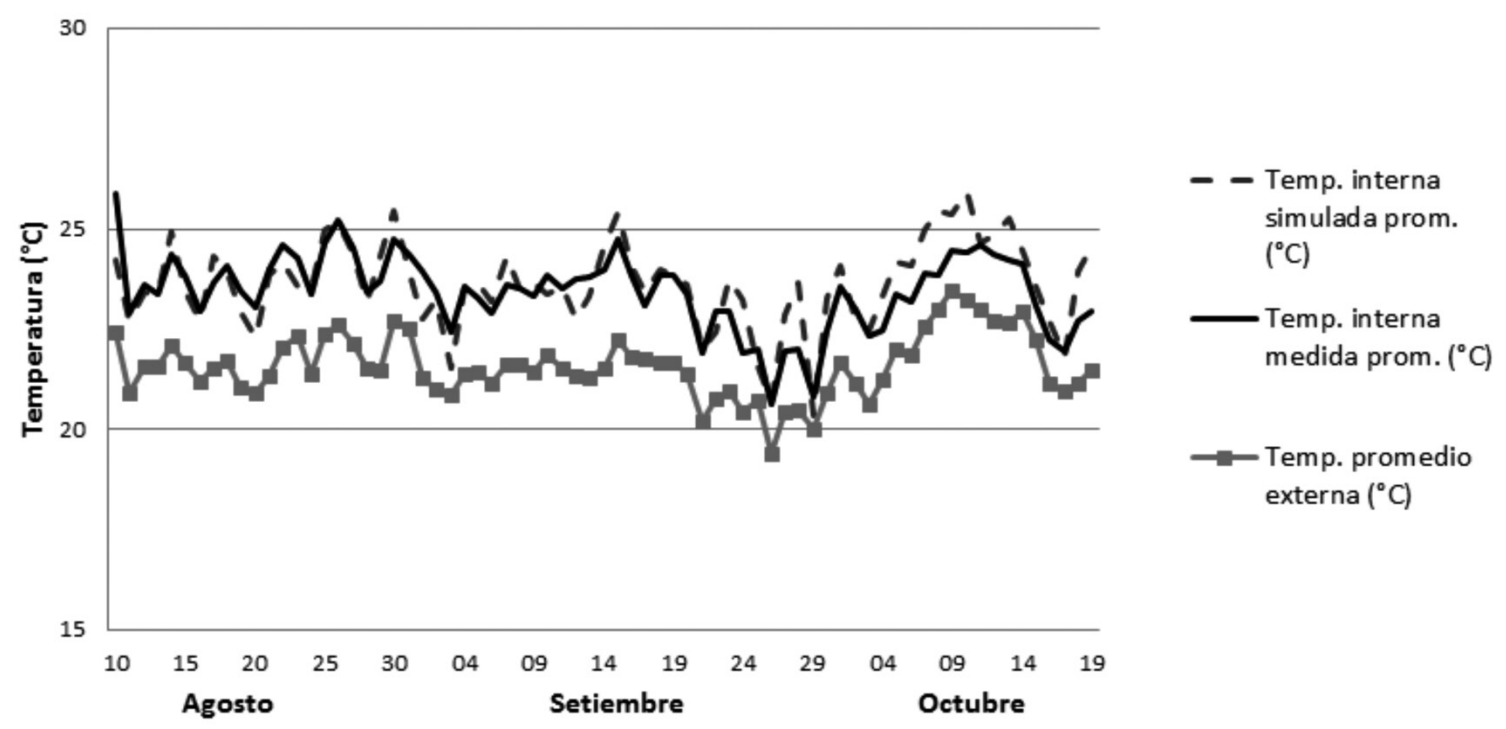

Figura 8. Temperaturas medidas y simuladas del invernadero de la Estación Experimental Agrícola Fabio Baudrit Moreno, en el mes de agosto a octubre del 2010. Alajuela, Costa Rica. Junio 2012. 
Cuadro 5. Raíz del error medio cuadrático relativo (RERMC).

\begin{tabular}{lc}
\hline Localización & $\begin{array}{c}\text { REMCR para temperatura } \\
\text { simulada }\end{array}$ \\
\hline Sicilia, Italia & 3,5 \\
Sicilia, Italia & 6,6 \\
Holanda & 6,2 \\
Holanda & 5,3 \\
Texas, USA & 6,0 \\
Texas, USA & 6,8 \\
Arizona, USA & 8 \\
Arizona, USA & 4,5 \\
Arizona, USA & 4,1 \\
Arizona, USA & 3 \\
\hline
\end{tabular}

Fuente: Vanthoor (2011).

\section{Resultados del balance energético}

En el Cuadro 6 se resumen los principales términos de energía que entran o salen del volumen de control de los distintos casos analizados aplicando la
Ecuación 1. Los valores aparecen en unidades de W/ $\mathrm{m}^{2} \mathrm{y}$ al final de cada caso se obtiene un valor en $\mathrm{KW}$, que corresponde a la cantidad de calor que debería retirarse, para poder obtener la temperatura objetivo establecida.

Se observó que el escenario más desfavorable es el F, en el cual se considera la temperatura máxima absoluta del mes más caliente (abril), y se intenta mantener una temperatura interna del invernadero de $25^{\circ} \mathrm{C}$ con solo un $20 \%$ cultivado. Este caso representa una situación extrema que puede darse. Se obtuvo una gran diferencia de temperatura entre el interior y el exterior. El caso $\mathrm{H}$ es de importancia para el diseño de sistemas de enfriamiento, porque toma en cuenta el promedio de las temperaturas máximas menos dos desviaciones estándar. Removiendo el calor que se genera con esta temperatura el invernadero operaría entre los rangos óptimos en un 95\% del tiempo aún con un 20\% del área cultivada.

También se observó el aumento de calor a retirar, como consecuencia de la disminución de porcentaje de área cultivada del invernadero. Entre mayor área cultivada tenga el invernadero, menos diferencia de temperatura habrá entre el volumen de control interno y el ambiente, este resultado concuerda con lo expuesto por Hellickson (1983), donde se demuestra el efecto refrigerante de la transpiración del cultivo. Este factor está relacionado con la pérdida de calor sensible que se

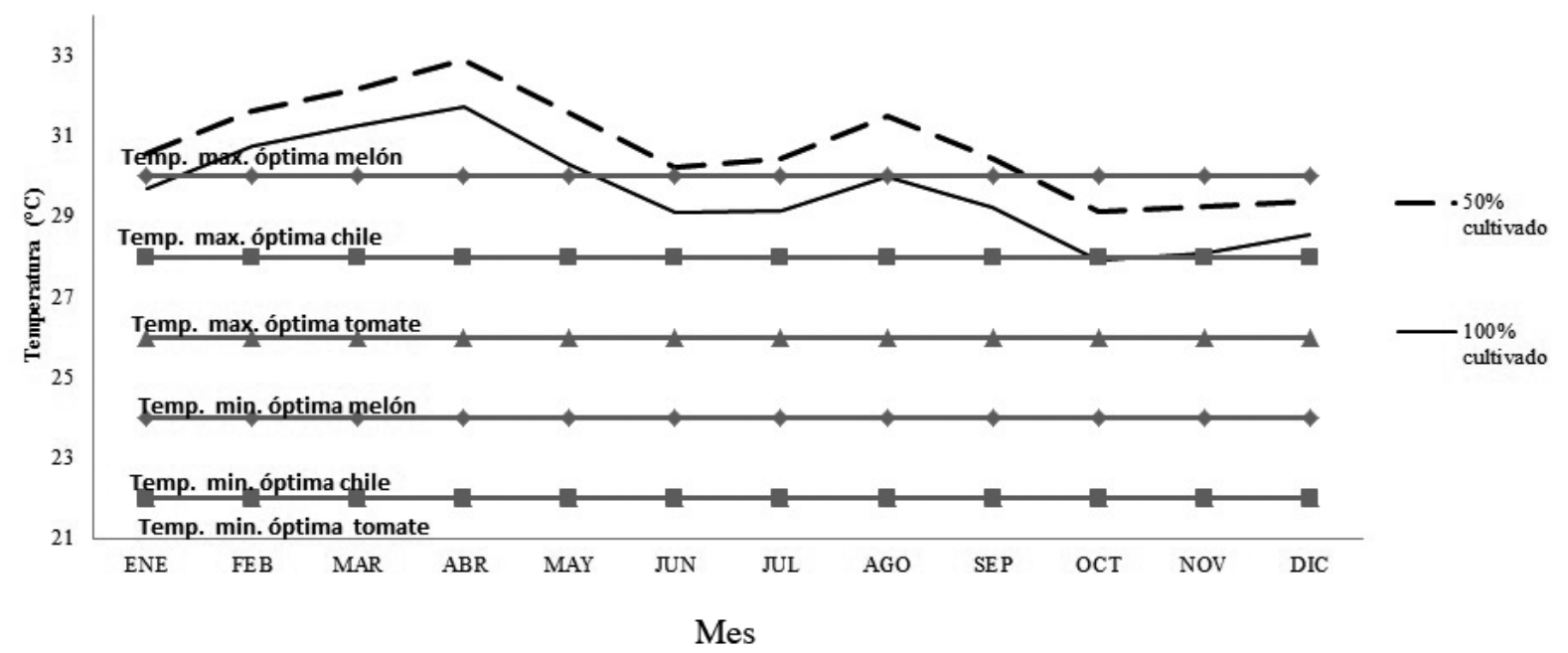

Figura 9. Temperaturas internas simuladas del invernadero de la Estación Experimental Agrícola Fabio Baudrit Moreno, utilizando promedios de temperaturas anuales IMN (2012) y factores de cultivo de 0,5 y 1. Alajuela, Costa Rica. Junio 2012. 
Cuadro 6. Resultado de los balances energéticos para diferentes condiciones climáticas y de cultivo en el invernadero de la Estación Experimental Agrícola Fabio Baudrit Moreno. Alajuela, Costa Rica. Julio 2012.*

\begin{tabular}{lcccccccccc}
\hline Casos & $\begin{array}{c}\text { Área } \\
\text { cultivada } \\
(\%)\end{array}$ & $\begin{array}{c}\text { Temp. } \\
\text { interna } \\
\text { objetivo } \\
\left({ }^{\mathbf{C}}\right)\end{array}$ & $\begin{array}{c}\text { Temp. } \\
\text { externa } \\
\left({ }^{\circ} \mathbf{C}\right)\end{array}$ & $\begin{array}{c}\mathbf{Q}_{\text {so }} \\
\left(\mathbf{W} / \mathbf{m}^{2}\right)\end{array}$ & $\begin{array}{c}\mathbf{Q}_{\mathbf{v}} \\
\left(\mathbf{W} / \mathbf{m}^{2}\right)\end{array}$ & $\begin{array}{c}\mathbf{Q}_{\mathrm{ce}} \\
\left(\mathbf{W} / \mathbf{m}^{2}\right)\end{array}$ & $\begin{array}{c}\mathbf{Q}_{\mathrm{tt}} \\
\left(\mathbf{W} / \mathbf{m}^{2}\right)\end{array}$ & $\begin{array}{c}\mathbf{Q}_{\mathrm{sl}} \\
\left(\mathbf{W} / \mathbf{m}^{2}\right)\end{array}$ & $\begin{array}{c}\text { Calor a } \\
\text { retirar, } \\
\mathbf{W} / \mathbf{m}^{2}\end{array}$ & $\begin{array}{c}\text { Calor a } \\
\text { retirar, } \\
\mathbf{K W}\end{array}$ \\
\hline $\mathbf{A}$ & 20 & 23 & 27,91 & 455,5 & 311,5 & 39,03 & $-36,5$ & $-45,55$ & 712,2 & 1504,17 \\
$\mathbf{B}$ & 50 & 23 & 31,19 & 455,5 & 511,3 & 65,11 & $-18,9$ & $-113,9$ & 887,8 & 1875,03 \\
$\mathbf{C}$ & 20 & 25 & 30,11 & 508,7 & 497 & 40,62 & $-38,7$ & $-50,87$ & 943,5 & 1992,67 \\
$\mathbf{D}$ & 50 & 25 & 30,11 & 508,7 & 497 & 40,62 & $-38,7$ & $-127,2$ & 867,2 & 1831,53 \\
$\mathbf{E}$ & 50 & 25 & 27,91 & 455,5 & 184,6 & 23,13 & -45 & $-113,9$ & 492,4 & 1039,95 \\
$\mathbf{F}$ & 20 & 25 & 32,51 & 508,7 & 722,5 & 59,7 & $-25,7$ & $-50,87$ & 1201,3 & 2537,15 \\
$\mathbf{G}$ & 20 & 25 & 31,19 & 455,5 & 386,5 & 49,21 & $-27,4$ & $-45,55$ & 806,6 & 1703,54 \\
$\mathbf{H}$ & 20 & 25 & 27,19 & 508,7 & 215,7 & 17,4 & $-54,1$ & $-50,9$ & 623,4 & 1316,6 \\
\hline
\end{tabular}

* Nomenclatura establecida en la ecuación 1.

convierte en latente dentro del invernadero y que varía con respecto a la radiación y a la evapotranspiración del cultivo.

Debe analizarse si equipando el invernadero con diferentes sistemas de enfriamiento y control de radiación, es posible controlar la temperatura y la humedad relativa de manera que la instalación sea viable para el cultivo de más productos a lo largo de todo el año.

$\mathrm{Si}$ se requiere la construcción de invernaderos para la experimentación, es más recomendable establecer módulos independientes con áreas pequeñas, similares a lo requerido por los lotes de la experimentación, de esta manera es más viable manejar las condiciones de clima óptimas de cada cultivo y evita además contaminaciones.

\section{LITERATURA CITADA}

Adams, S.R., K.E. Cockshull, y C.R.J. Cave. 2001. Effect of temperature on the growth and development of tomato fruits. Annals of Botany 88(5):869-877.

Alas, M. 2003. Estructura de costos, para la producción de hortalizas en invernaderos de la cuenca del Río Reventazón, Turrialba, Costa Rica. Tesis de Mag. Sc., CATIE, Turrilaba, Costa Rica.
Albright, L.D. 1990. Environment control for animals and plants. ASAE, St. Joseph, MO, USA.

Barquero, G. 2001. Producción en ambiente controlado. Colegio de Ingenieros Agrónomos, San José, Costa Rica.

Castellanos, J.Z. 2009. Manual de producción de tomate en invernadero. Intagri, Celaya, Guanajuato, México.

Costa, E., P. Leal, y R. Carmo. 2004. Modelo de simulação da temperatura e umidade relativa do ar no interior de estufa plástica. Eng. Agríc., Jaboticabal 24(1):5767.

Hellickson, M. 1983. Ventilation of agricultural structures. American Society of Agricultural Engineers, St. Joseph, Missouri, USA.

IMN (Instituto Meteorológico Nacional). 2012. Información meteorológica de la estación climática. Series horarias correspondiente al periodo de mayo de 2009 a octubre 2011. Estación Experimental Fabio Baudrit Moreno, Universidad de Costa Rica, Alajuela, Costa Rica.

Kittas, C., T. Bartzanas, y A. Jaffrin. 2003. Temperature gradients in a partially shaded large greenhouse equipped with evaporative cooling pads. Biosystems Engineering 85:87-94.

Leal, P., y E. Costa. 2011. Apostilla de ingeniería de confort en cultivo protegido. Universidade Estadual de Campinas, Campinas, Brasil.

López, A.J. 2013. Validación de un modelo matemático para predecir las condiciones climáticas internas en 
un invernadero localizado en la zona norte de Cartago, Costa Rica. Tesis de Lic., Universidad de Costa Rica, San José, Costa Rica.

Montero, J. 2006. Evaporative cooling in greenhouses: effect on microclimate, water use in efficiency and plant response. Acta Hort. 719:373-384.

Pete, M.M., D.H. Willits, y R. Gardner. 1997. Response of ovule development and postpollen production processes in male-sterile tomatoes to chronic, subacute high temperature stress. Journal of Experimental Botany 48(306):101-111

Piscia, D., J.I. Montero, M. Melé, J. Flores, J. Perez-Parra, y E.J. Baeza. 2012. A CFD model to study above roof shade and on roof shade of greenhouses. Dept. of Environmental Horticulture. IRTA, Cabrils Barcelona. Estación Experimental de Cajamar "Las Palmerillas", El Ejido Almería España.
Serrano, Z. 1994. Construcción de invernaderos. MundiPrensa, Madrid, España.

Stanghellini, C. 1987. Transpiration of greenhouse crops; an aid to climate management. Ph. D. Dissertation, Landbouwuniverseit, Wageningen, The Netherland.

Tesi, R. 2001. Medios de protección para la hortoflorofruticultura y el viverismo. 3 ed. MundiPrensa, Madrid, España.

Vanthoor, B. 2011. A model-based greenhouse design method. Ph.D. Dissertation, Wageningen University, The Netherlands.

Zhang J., T. Li, y J. Xu. 2008. Effects of sub-high temperature in daytime from different stages on tomato photosynthesis and yield in greenhouse. Nongye Gongcheng Xuebao/Transactions of the Chinese Society of Agricultural Engineering 24(3):193-197. 